\title{
Modeling the Motion of a Flapping Wing Aerial Vehicle
}

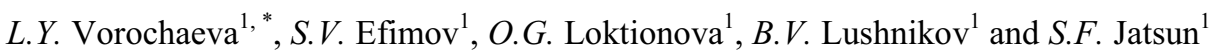 \\ ${ }^{1}$ Southwest State University, 305040 Kursk, Russia
}

\begin{abstract}
The article discusses the vertical flight of a flapping wing aerial vehicle, which is also called an ornithopter. The robot is a chain of five links connected in series by active cylindrical hinges with the central link being the body and the remainder forming folding wings in pairs. The distinctive feature of this device is that the flaps of its wings imitate those of a seagull i.e. the device has a biological prototype. We construct a mathematical model of this device; much attention is given to the model of the interaction of the wings with the air environment and we determine the positions and velocities of points of application of the reduced aerodynamic forces to each of the links. Based on the results of numerical modelling of the vertical flight of the robot three modes of flight were established: ascent, hovering at a certain height and descent. The device can operate in these modes based on the oscillation parameters of the wings in particular flapping frequency and amplitude, the ratio of the amplitudes of two links and one wing and the shift of the equilibrium oscillation position of the wings relative to zero.
\end{abstract}

\section{Introduction}

With the development of robotics and related technologies more and more interest is shown towards bionics - the science that studies the nature of the movement of living organisms, as well as the phenomena and processes taking place in them in order to create mechatronic devices operating on the same principles.

The study of the character of the movement of birds has enabled us to makes an important conclusion that a system of three or five rigid bodies connected by hinges equipped with electric drives can be used as a simplified system that adequately models the motion of a robotornithopter [1-7]. By feeding control voltages to the actuators we can change the relative angles defining the position of the links with respect to each other by given values.

\section{Description of the ornithopter}

The article discusses a flapping wing device called an ornithopter, which consists of a chain of five links [5]. Each wing of the robot is formed by two links: 1 and 2, 4 and 5 , connected to each other by cylindrical hinges that also connect them with the body 3 (fig. 1). The cylindrical hinges are active due to actuators 6 that create the flapping motion of the wings relative to the body. The distinctive feature of this work is that the horizontal projection of the area of the wings of the device changes in each flap, which results in flight: the wings fold during the upward flap causing a reduction in aerodynamic resistance on the wings and unfold during the downward flap causing an increase in the in aerodynamic resistance under the wings.

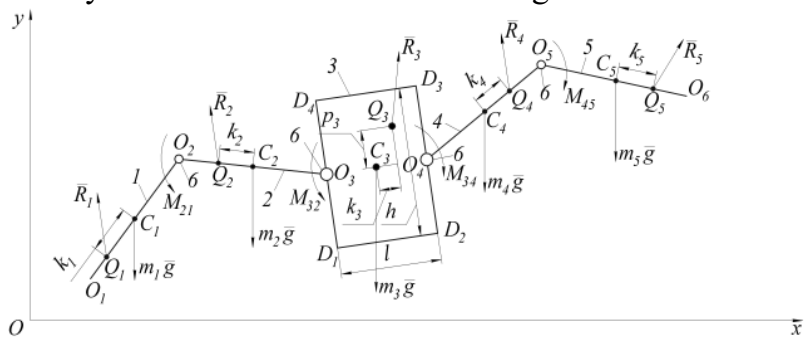

Fig. 1. Settlement scheme of the ornithopter.

The work studies the motion of an ornithopter in the vertical plane $O x y$ under the condition that the body of the device is modeled by a rectangle with sides $l_{3}$ and $h_{3}$ and the links - by rods of length $l_{i}$. We also assume that the centres of mass of the links coincide with their symmetry points $-C_{i}$. The relative coordinate system $O_{i} x_{i} y_{i}$ is associated with each link such that the axis $O_{i} x_{i}$ is located along the link (for link 3 the relative coordinate system $C_{3} x_{3} y_{3}$ the axis $C_{3} x_{3}$ passes through the points $C_{3}$ and $O_{4}$ ). The position of the robot in the Oxy plane is uniquely described by the projections of the position of the body's center of mass on axes $O x$ and $O y$ and the angles of inclination of the links to the horizontal plane $\varphi_{i}$, which are measured in the anticlockwise direction. Thus the general coordinates vector has the form

$$
\bar{q}=\left(\begin{array}{lllllll}
x_{C 3} & y_{C 3} & \varphi_{1} & \varphi_{2} & \varphi_{3} & \varphi_{4} & \varphi_{5}
\end{array}\right)^{T} .
$$

The flight of the ornithopter takes place under the action of gravitational forces, $m_{i} g$ acting at the centers of 
mass of the links, moments $M_{21}, M_{32}, M_{34}$ and $M_{45}$ generated by the actuators and aerodynamic forces $R_{i}$ applied at points $Q_{i}$.

\section{Aerodynamic model}

\subsection{Determination of aerodynamic forces}

During the motion of the ornithopter aerodynamic forces act on every link $i=1-5$. These forces can be written as components on the absolute and relative coordinate axes as follows (fig. 2):

$$
\bar{R}_{i}=\bar{R}_{i x}+\bar{R}_{i y}=\bar{R}_{i x}^{(i)}+\bar{R}_{i y}^{(i)}
$$

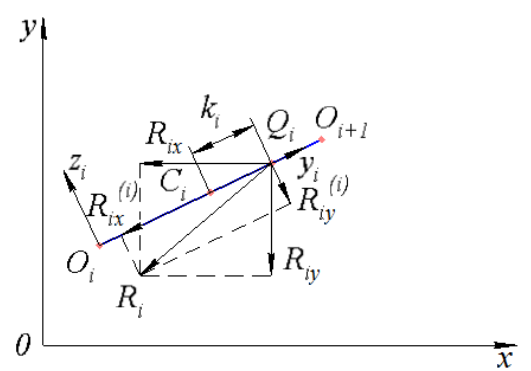

Fig. 2. Settlement scheme of aerodynamic forces on links $i=1$, $2,4,5$.

The component of aerodynamic force in the absolute coordinate system is given by the following formulas:

$$
R_{i z}=\frac{C_{x} \rho S_{i}^{O x}}{2} \dot{x}_{Q i}\left|\dot{x}_{Q i}\right| R_{i y}=\frac{C_{y} \rho S_{i}^{O_{y}}}{2} \dot{y}_{Q i}\left|\dot{y}_{Q i}\right|,
$$

where $C_{x}, C_{y}$ - coefficients of the aerodynamic force components, $S_{i}^{O x}$ and $S_{i}^{O y}$ - areas of sides of $i$-th link perpendicular to axes $O x$ and $O y$ respectively, $\dot{x}_{Q i}, \dot{y}_{Q i}$ - velocity components of points $Q_{i}$ on axes $O x$ and $O y$.

We calculate areas $S_{i}^{O x}$ and $S_{i}^{O_{y}}$ by the formulas:

$$
\begin{aligned}
& S_{i}^{O x}=S_{i}^{\text {Oixi }} \cos \varphi_{i}+S_{i}^{\text {Oiyi }} \sin \varphi_{i} \\
& S_{i}^{O y}=S_{i}^{\text {Oixi }} \sin \varphi_{i}+S_{i}^{\text {Oiyi }} \cos \varphi_{i}
\end{aligned}
$$

where

$$
S_{i}^{\text {Oixi }}=b_{i} h_{i}, S_{i}^{\text {Oiyi }}=l_{i} b_{i}
$$

- areas of the links peprendicular to axes $O_{i} x_{i}$ and $O_{i} y_{i}$ of the relaive coordinates systems for $i=1,2,4,5 S_{i}^{\text {Oixi }}=0$, $b_{i}$ - the width of the links (not shown in fig. 1) determined in the direction perpendicular to plane $O x y$.

In order to determine the positions of points $Q_{i}$ on the links we assume that points $Q_{i}$ are the centers of mass of the figure formed by forces $\bar{R}_{i y}^{(i)}$ acting peperndicular $\mathrm{t}$ the links. The links are located at distances of $k_{i}$ from the centers of mass of the links. The position of point $Q_{3}$ is determined by the destances, $k_{3}$ and $p_{3}$ relative to the center of mass of the latter along sides $D_{1} D_{2}$ and $D_{2} D_{3}$.

\subsection{Determination of the velocities of the points $\boldsymbol{Q}_{i}$}

The velocities of the points $Q_{i}$ are determined from their positions on the links given earlier, which constantly change during the robot's motion. The velocities of points $Q_{2}$ and $Q_{4}$ equal:

$$
\begin{aligned}
& \dot{\vec{r}}_{Q 2}=\dot{\vec{r}}_{C 3}+\dot{T}_{30} \vec{\rho}_{C 3 O 3}^{(3)}+\dot{T}_{20}\left(\vec{\rho}_{O 3 C 2}^{(2)}+\vec{\rho}_{C 2 Q 2}^{(2)}\right), \\
& \dot{\vec{r}}_{Q 4}=\dot{\vec{r}}_{C 3}+\dot{T}_{30} \vec{\rho}_{C 3 O 4}^{(3)}+\dot{T}_{40}\left(\vec{\rho}_{O 4 C 4}^{(4)}+\vec{\rho}_{C 4 Q 4}^{(4)}\right)
\end{aligned}
$$

where

$$
\vec{\rho}_{C 2 Q 2}^{(2)}=\left(\begin{array}{ll}
k_{2} & 0
\end{array}\right)^{T}, \vec{\rho}_{C 4 Q 4}^{(4)}=\left(\begin{array}{ll}
k_{4} & 0
\end{array}\right)^{T} \text { - the corresponding }
$$
relative radius-vectors, $\dot{T}_{i 0}-$ the derivative of the rotation matrix

$$
T_{i 0}=\left(\begin{array}{cc}
\cos \varphi_{i} & -\sin \varphi_{i} \\
\sin \varphi_{i} & \cos \varphi_{i}
\end{array}\right)
$$

The velocities of points $Q_{1}, Q_{5}$ equal:

$$
\begin{aligned}
\dot{\vec{r}}_{Q 1}= & \dot{\vec{r}}_{C 3}+\dot{T}_{30} \vec{\rho}_{C 3 O 3}^{(3)}+\dot{T}_{20} \vec{\rho}_{O 3 O 2}^{(2)}+ \\
& +\dot{T}_{10}\left(\vec{\rho}_{O 2 C 1}^{(1)}+\vec{\rho}_{C 1 Q 1}^{(1)}\right) \\
\dot{\vec{r}}_{Q 5}= & \dot{\vec{r}}_{C 3}+\dot{T}_{30} \vec{\rho}_{C 3 O 4}^{(3)}+\dot{T}_{40} \vec{\rho}_{O 4 O 5}^{(4)}+ \\
& +\dot{T}_{50}\left(\vec{\rho}_{O 5 C 5}^{(5)}+\vec{\rho}_{C 5 Q 5}^{(5)}\right)
\end{aligned}
$$

where $\quad \vec{\rho}_{C 1 Q 1}^{(2)}=\left(\begin{array}{ll}k_{1} & 0\end{array}\right)^{T}, \quad \vec{\rho}_{C 5 Q 5}^{(5)}=\left(\begin{array}{ll}k_{5} & 0\end{array}\right)^{T}-\quad$ the corresponding relative radius-vectors.

The velocity of point $Q_{3}$ has the form:

$$
\dot{\vec{r}}_{Q 3}=\dot{\vec{r}}_{C 3}+\dot{T}_{30} \vec{\rho}_{C 3 Q 3}^{(3)} \text {. }
$$

where $\vec{\rho}_{C 3 Q 3}^{(3)}=\left(\begin{array}{ll}k_{3} & p_{3}\end{array}\right)^{T}$ - the relative radius-vector.

\subsection{Determination of the positions of points $Q_{i}$}

In order to determine the locations of points $Q_{i}$ on the links (distances $k_{i}$ and $p_{3}$ ) we consider the corresponding aerodynamic force components $R_{i y}^{(i)}$ and $R_{3 x}^{(3)}$, perpendicular to axes $O_{i} x_{i}$ and $O_{3} y_{3}$ and assume points $Q_{i}$ to be the cemters of mass of the corresponding figures. The velocity components of points $Q_{i}$ in the $O_{i} x_{i} y_{i}$ cordinate system are writtend using the rotation matrix $T_{0 i}$ :

$$
\begin{array}{r}
\dot{r}_{Q i}^{(i)}=T_{0 i} \dot{r}_{Q i} \\
T_{0 i}=T_{i 0}^{-1}=\left(\begin{array}{cc}
\cos \varphi_{i} & \sin \varphi_{i} \\
-\sin \varphi_{i} & \cos \varphi_{i}
\end{array}\right) .
\end{array}
$$


We move on to evaluate the distances $k_{i}$. For this purpose we determine the formulas for $\left(\dot{y}_{Q i}^{(i)}\right)^{2}$ by grouping the terms before $k_{i}^{2}, k_{i}, k_{i}^{0}$ respectively and obtain formulars for calculating the components of aerodynamic forces on axes $O_{i} y_{i}$. We present $R_{i y}^{(i)}$ as follows

$$
R_{i y}^{(i)}=a_{i y} k_{i}^{2}+b_{i y} k_{i}+c_{i y} .
$$

We introduce the denotations below.

$$
\begin{aligned}
& a_{i y}=\frac{C_{y} \rho S_{i}^{O i y i}}{2} \dot{\varphi}_{i}^{2} \\
& b_{i=1,5 y}=\frac{C_{y} \rho S_{i}^{\text {Oiyi }}}{2}\left[-2 \dot{x}_{C 3} \dot{\varphi}_{i} \sin \varphi_{i}\right. \\
& +2 \dot{y}_{C 3} \dot{\varphi}_{i} \cos \varphi_{i} \mp \dot{\varphi}_{i} \dot{\varphi}_{3} l_{3} \cos \left(\varphi_{3}-\varphi_{i}\right) \mp \\
& \left.\mp 2 \dot{\varphi}_{i} \dot{\varphi}_{2(4)} l_{2(4)} \cos \left(\varphi_{2(4)}-\varphi_{i}\right) \mp \dot{\varphi}_{i}^{2} l_{i}\right] \\
& b_{i=2,4 y}=\frac{C_{y} \rho S_{i}^{O i y i}}{2}\left[-2 \dot{x}_{C 3} \dot{\varphi}_{i} \sin \varphi_{i}+\right. \\
& \left.+2 \dot{y}_{C 3} \dot{\varphi}_{i} \cos \varphi_{i} \mp \dot{\varphi}_{i} \dot{\varphi}_{3} l_{3} \cos \left(\varphi_{3}-\varphi_{i}\right) \mp \dot{\varphi}_{i}^{2} l_{i}\right] \\
& b_{3 y}=-C_{y} \rho S_{3}^{O 3 y 3} \dot{\varphi}_{3}\left(\dot{y}_{C 3} \cos \varphi_{3}-\dot{x}_{C 3} \sin \varphi_{3}\right) \\
& c_{i=1,5 y}=\frac{C_{y} \rho S_{i}^{O i y i}}{2}\left[\dot{y}_{C 3}^{2} \sin ^{2} \varphi_{i}-\right. \\
& 2 \dot{x}_{C 3} \dot{y}_{C 3} \sin \varphi_{i} \cos \varphi_{i}+\dot{y}_{C 3}^{2} \cos ^{2} \varphi_{i} \pm \\
& \pm \dot{x}_{C 3} \dot{\varphi}_{3} l_{3} \sin \varphi_{i} \cos \left(\varphi_{3}-\varphi_{i 1}\right) \pm \\
& 2 \dot{x}_{C 3} \dot{\varphi}_{i \pm 1} l_{i \pm 1} \sin \varphi_{i} \cos \left(\varphi_{i \pm 1}-\varphi_{i}\right) \pm \dot{x}_{C 3} \dot{\varphi}_{i} l_{i} \sin \varphi_{i} \mp \\
& \mp \dot{y}_{C 3} \dot{\varphi}_{3} l_{3} \cos \varphi_{i} \cos \left(\varphi_{3}-\varphi_{i}\right) \mp \\
& 2 \dot{y}_{C 3} \dot{\varphi}_{i \pm 1} l_{i \pm 1} \cos \varphi_{i} \cos \left(\varphi_{i \pm 1}-\varphi_{i}\right) \mp \dot{y}_{C 3} \dot{\varphi}_{i} l_{i} \cos \varphi_{i}+ \\
& +\dot{\varphi}_{3}^{2} l_{3}^{2} \cos ^{2}\left(\varphi_{3}-\varphi_{i}\right) / 4+ \\
& \dot{\varphi}_{i \pm 1} \dot{\varphi}_{3} l_{i \pm 1} l_{3} \cos \left(\varphi_{i \pm 1}-\varphi_{i}\right) \cos \left(\varphi_{3}-\varphi_{i}\right)+ \\
& \dot{\varphi}_{i} \dot{\varphi}_{3} l_{i} l_{3} \cos \left(\varphi_{3}-\varphi_{i}\right) / 2+ \\
& +\dot{\varphi}_{i \pm 1}^{2} l_{i \pm 1}^{2} \cos ^{2}\left(\varphi_{i \pm 1}-\varphi_{i}\right)+ \\
& \left.+\dot{\varphi}_{i} \dot{\varphi}_{i \pm 1} l_{i} l_{i \pm 1} \cos \left(\varphi_{i \pm 1}-\varphi_{i}\right)+\dot{\varphi}_{i}^{2} \frac{l_{i}^{2}}{4}\right] \\
& c_{i=2,4 y}=\frac{C_{y} \rho S_{i}^{O i y i}}{2}\left[\dot{x}_{C 3}^{2} \sin ^{2} \varphi_{i}-2 \dot{x}_{C 3} \dot{y}_{C 3} \sin \varphi_{i} \cos \varphi_{i}+\right. \\
& +\dot{y}_{C 3}^{2} \cos ^{2} \varphi_{i} \pm \dot{x}_{C 3} \dot{\varphi}_{3} l_{3} \sin \varphi_{i+1} \cos \left(\varphi_{3}-\varphi_{i}\right) \pm \\
& \dot{x}_{C 3} \dot{\varphi}_{i} l_{i} \sin \varphi_{i} \mp \dot{y}_{C 3} \dot{\varphi}_{3} l_{3} \cos \varphi_{i} \cos \left(\varphi_{3}-\varphi_{i}\right) \mp \dot{y}_{C 3} \dot{\varphi}_{i} l_{i} \cos \varphi_{i}+ \\
& \left.+\dot{\varphi}_{3}^{2} \frac{l_{3}^{2}}{4} \cos ^{2}\left(\varphi_{3}-\varphi_{i}\right)+\dot{\varphi}_{i} \dot{\varphi}_{3} l_{i} l_{3} \cos \left(\varphi_{3}-\varphi_{i}\right) / 2+\dot{\varphi}_{i}^{2} \frac{l_{i}^{2}}{4}\right]
\end{aligned}
$$

$$
\begin{aligned}
b_{3 x}= & -C_{x} \rho S_{3}^{O 3 x 3} \dot{\varphi}_{3}\left(\dot{x}_{C 3} \cos \varphi_{3}+\dot{y}_{C 3} \sin \varphi_{3}\right) \\
& c_{3 x}=\frac{C_{x} \rho S_{3}^{O 3 x 3}}{2}\left[\dot{x}_{C 3}^{2} \cos ^{2} \varphi_{3}\right. \\
& \left.+2 \dot{x}_{C 3} \dot{y}_{C 3} \sin \varphi_{3} \cos \varphi_{3}+\dot{y}_{C 3}^{2} \sin ^{2} \varphi_{3}\right]
\end{aligned}
$$

and write the force $R_{3 x}^{(3)}$ as follows

$$
R_{3 x}^{(3)}=a_{3 x} p_{3}^{2}+b_{3 x} p+c_{3 x}
$$

We find the distance $p_{3}$ according to the formula

$$
p_{3}=\frac{\int_{-h 3 / 2}^{h 3 / 2} p_{3} \cdot R_{3 x}^{(3)} d p_{3}}{\int_{-h 3 / 2}^{h . / 2} R_{3 x}^{(3)} d p_{3}}=\frac{b_{3 x} h_{3}{ }^{2}}{a_{3 x} h_{3}{ }^{2}+12 c_{3 x}}
$$

For the special case when the velocity of rotation, $\dot{\varphi}_{i} \rightarrow 0$, the expression for the application points of the aerodynamic force will have the form:

$$
\begin{gathered}
a_{3 x}\left(\dot{\varphi}_{3} \approx 0\right)=0, a_{i y}\left(\dot{\varphi}_{i} \approx 0\right)=0, i=1-5, \\
b_{3 x}\left(\dot{\varphi}_{3} \approx 0\right)=0, b_{i y}\left(\dot{\varphi}_{i} \approx 0\right)=0, i=1-5, \\
c_{3 x}\left(\dot{\varphi}_{3} \approx 0\right) \neq 0, c_{i y}\left(\dot{\varphi}_{i} \approx 0\right) \neq 0, i=1-5, \\
p_{3}=0, k_{i}=0 .
\end{gathered}
$$


Therefore we can conclude that for $\dot{\varphi}_{i} \approx 0$ the points of application of the aerodynamic force on links $i=1-5$ will be located at their centers of mass.

\section{Mathematical Model for the Flight of the Ornithopter}

The system of differential equations of the ornithopter's motion written using the $2^{\text {nd }}$ order Lagrange equation and the virtual work principle can be presented as follows:

$$
A(q) \ddot{q}+B(q) D(\dot{q}) \dot{q}=F
$$

where $A(q), B(q)$ are matrices of coefficients, $D(\dot{q})-$ the diagonal matrix of the first derivatives of the general coordinates $q$ and $F$ - the matrix of generalised forces

$$
A(q)=\left(\begin{array}{ccccccc}
a_{11} & 0 & a_{13} & a_{14} & a_{15} & a_{16} & a_{17} \\
0 & a_{22} & a_{23} & a_{24} & a_{25} & a_{26} & a_{27} \\
a_{31} & a_{32} & a_{33} & a_{34} & a_{35} & 0 & 0 \\
a_{41} & a_{42} & a_{43} & a_{44} & a_{45} & 0 & 0 \\
a_{51} & a_{52} & a_{53} & a_{54} & a_{55} & a_{56} & a_{57} \\
a_{61} & a_{62} & 0 & 0 & a_{65} & a_{66} & a_{67} \\
a_{71} & a_{72} & 0 & 0 & a_{75} & a_{76} & a_{77}
\end{array}\right),
$$

$$
B(q)=\left(\begin{array}{ccccccc}
0 & 0 & b_{13} & b_{14} & b_{15} & b_{16} & b_{17} \\
0 & 0 & b_{23} & b_{24} & b_{25} & b_{26} & b_{27} \\
0 & 0 & 0 & b_{34} & b_{35} & 0 & 0 \\
0 & 0 & b_{43} & 0 & b_{45} & 0 & 0 \\
0 & 0 & b_{53} & b_{54} & 0 & b_{56} & b_{57} \\
0 & 0 & 0 & 0 & b_{65} & 0 & b_{67} \\
0 & 0 & 0 & 0 & b_{75} & b_{76} & 0
\end{array}\right),
$$

$$
\begin{gathered}
D(\dot{q})=\left(\begin{array}{ccccccc}
\dot{q}_{1} & 0 & 0 & 0 & 0 & 0 & 0 \\
0 & \dot{q}_{2} & 0 & 0 & 0 & 0 & 0 \\
0 & 0 & \dot{q}_{3} & 0 & 0 & 0 & 0 \\
0 & 0 & 0 & \dot{q}_{4} & 0 & 0 & 0 \\
0 & 0 & 0 & 0 & \dot{q}_{5} & 0 & 0 \\
0 & 0 & 0 & 0 & 0 & \dot{q}_{6} & 0 \\
0 & 0 & 0 & 0 & 0 & 0 & \dot{q}_{7}
\end{array}\right), \\
F=\left(\begin{array}{lllllll}
F_{1} & F_{2} & F_{3} & F_{4} & F_{5} & F_{6} & F_{7}
\end{array}\right)^{T}
\end{gathered}
$$

\section{Modeling of Vertical Flight of the Ornithopter}

The analytical diagram of the ornithopter proposed in this work allows the device to imitate the flapping motion of birds and consequently, their behaviour during flight. In this connection, we constructed the laws of variation of the angles of the wing links, for which the behavior of the device is analogous to that of seagull [5].

$$
\begin{aligned}
& \varphi_{2}(t)=-\varphi_{02} \cos (\omega t)-w_{2} \\
& \varphi_{4}(t)=\varphi_{04} \cos (\omega t)+w_{4} \\
& \varphi_{1}(t)=\left\{\begin{array}{l}
\varphi_{01} \cos \left(\omega_{1} t\right)+w_{1}, t<\frac{T}{4} \\
-\varphi_{01} \cos \left(\omega\left(t-\frac{T}{4}\right)\right)+w_{1}, \frac{T}{4} \leq t<\frac{3 T}{4} \\
\varphi_{01}+w_{1}, \frac{3 T}{4} \leq t \leq T
\end{array}\right. \\
& \varphi_{5}(t)=\left\{\begin{array}{l}
-\varphi_{05} \cos \left(\omega_{1} t\right)-w_{5}, t<\frac{T}{4} \\
\varphi_{05} \cos \left(\omega\left(t-\frac{T}{4}\right)\right)-w_{5}, \frac{T}{4} \leq t<\frac{3 T}{4} \\
-\varphi_{05}-w_{5}, \frac{3 T}{4} \leq t \leq T
\end{array}\right.
\end{aligned}
$$

where $\varphi_{0 i}$ is a certain value of the rotation angle of the link that determines the amplitude of the oscillations, $w_{i}$ is the displacement of the equillibrium position of the link's oscillation upwards/downwards, $T$ - the period of oscillations of the link. We have $\varphi_{05}^{-}=-\varphi_{05}-w_{5}$, $\varphi_{05}^{+}=\varphi_{05}-w_{5}, \varphi_{04}^{-}=-\varphi_{04}+w_{4}, \varphi_{04}^{+}=\varphi_{04}+w_{4}$.

We introduce the following denotations: $\varphi_{01}=\varphi_{05}=\varphi_{0}^{\prime}, \quad \varphi_{02}=\varphi_{04}=\varphi_{0}, \quad w_{1}=w_{2}=w_{4}=w_{5}=w$ and assume that $\omega_{1}=2 \omega$. We introduce a coefficient characterising the ration of the oscillation amplitudes of links 4 and 5 (1 and 2)

$$
\lambda=\frac{\varphi_{04}}{\varphi_{05}}=\frac{\varphi_{0}}{\varphi_{0}^{\prime}} .
$$

The time graphs of the coordinate of the body's center of mass, $y_{C 3}$ for different values of the displacement ratios $w_{4}=-w_{5}$ are shown in fig. 3. It can be seen from the graphs that there are three modes of vertical flight of the device: ascent, hovering and descent. As the ratios $w_{4}=-$ $w_{5}$ increase the descent mode switches to hovering, then ascent. For negative values of $w_{4}=-w_{5}$ the distance descended by the ornithopter decreases as the ratios approach 0 . The distance by which the device ascends also decreases for positive values of $w_{4}=-w_{5}$ as the ratios approach $0 \mid$.

It is more convenient to analyse the dependence of the vertical speed of the device on the flapping amplitude of the wings for different values of oscillation parameters. The average speeds of motion of the body's centre of mass, $\dot{y}_{s r}$ for different modes of motion are presented in figs. 4-6. The average speed is given by the formula

$$
\dot{y}_{s r}=1 / N \sum_{j=1-N} \dot{y}_{C 3},
$$


where $N$ is the number of calculations for one period of wing oscillation.
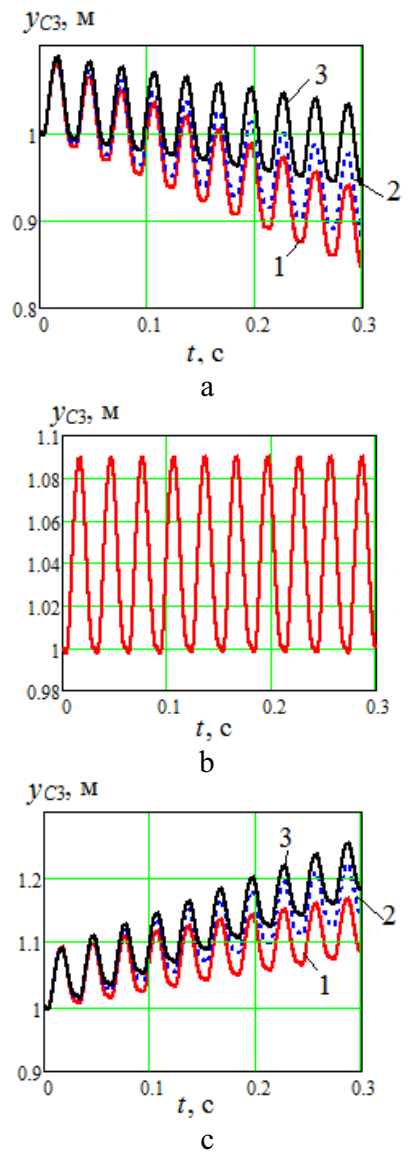

Fig. 3. Graphs of $y_{C 3}(t)$ for $T=0.03 \mathrm{~s}, \varphi_{0}=0.4 \mathrm{rad}$ : a - descent $\left(1-w_{4}=-w_{5}=-15^{0}, 2-w_{4}=-w_{5}=-10^{0}, 3-w_{4}=-w_{5}=-5^{0}\right)$, $\mathrm{b}$ - hovering $\left(w_{4}=-w_{5}=-1^{0}\right), \mathrm{c}$ - ascent $\left(1-w_{4}=-w_{5}=5^{0}\right.$, $\left.2-w_{4}=-w_{5}=10^{0}, 3-w_{4}=-w_{5}=15^{0}\right)$.

It can be seen from the graphs in fig. 4 that as the frequency, $\omega$ decreases (increase in the period of oscillations, $T$ ) the values of the average speeds decrease and a maximum is observed for all curves, which is evened-out as $\omega$ decreases.

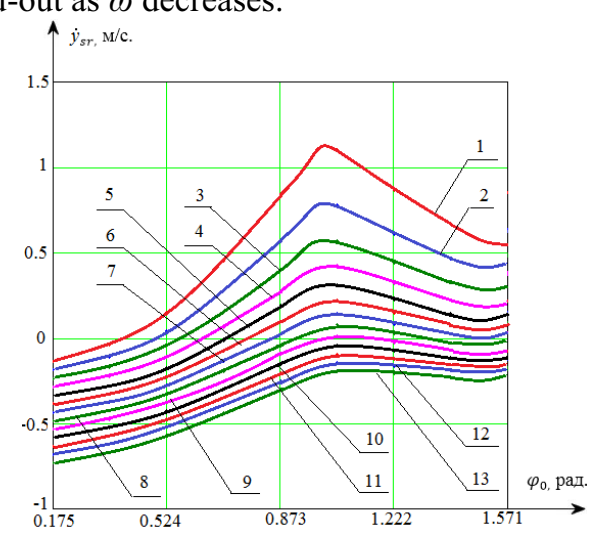

Fig. 4. Graphs of : $1-T=0,03 \mathrm{~s}, 2-T=0,04 \mathrm{~s}, 3-T=0,05 \mathrm{~s}$,

$4-T=0,06 \mathrm{~s}, 5-T=0,07 \mathrm{~s}, 6-T=0,08 \mathrm{~s}, 7-T=0,09 \mathrm{~s}, 8-$ $T=0,10 \mathrm{~s}, 9-T=0,11 \mathrm{~s}, 10-T=0,12 \mathrm{~s}, 11-T=0,13 \mathrm{~s}, 12-$ $T=0,14 \mathrm{~s}, 13-T=0,15 \mathrm{~s}$.
At the smallest angle, $\varphi_{0}=0.175 \mathrm{rad}$ the values of the average velocities are negative and at the greatest angle, $\varphi_{0}=1.571-$ negative for small oscillation frequencies and positive for large values of $\omega$. This means that for large frequencies the following alternation of the modes of motion occurs depening on the increase in the amplitude of oscillations: descent, hovering and ascent. However, when the frequencies decrease the alternation sequence of the modes of motion is different: descent, hovering, ascent, hovering, descent and there is only one mode of motion at the smallest frequencies.

Fig. 5 shows the results of the study of the influence of the amplitude ratio of the oscillation amplitude of the links on the average values of the velocity of vertical displacement of the device.
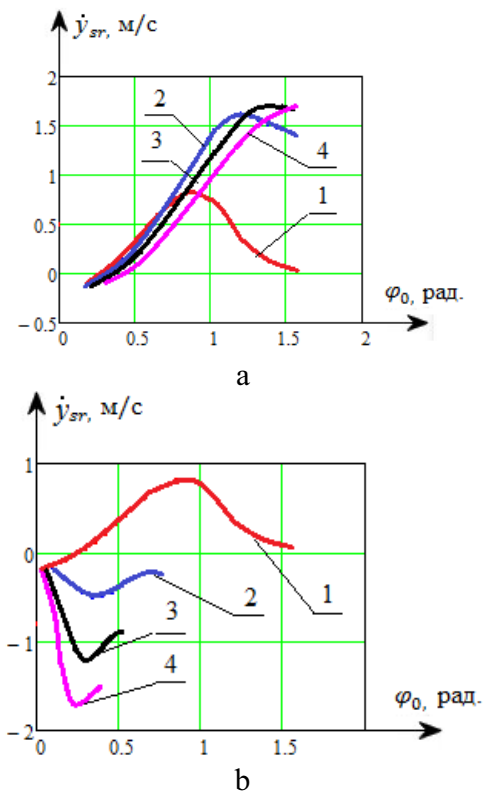

Fig. 5. Graphs of $\dot{y}_{s r}\left(\varphi_{0}\right)$ for $w=0 \mathrm{rad}, T=0,03 \mathrm{~s}: \mathrm{a}-1-\lambda=1$, $2-\lambda=2,3-\lambda=3,4-\lambda=4, b-1-\lambda=1,2-\lambda=1 / 2,3-\lambda=1 / 3$, $4-\lambda=1 / 4$

We can single out three types of curves from the given graphs. The first type holds for the amplitude $\lambda=1$ when the average velocity $\dot{y}_{s r}$ is negative and close to zero at the minimum value of $\varphi_{0}$. As $\varphi_{0}$ increases up to the value, $\varphi_{0}=0.785 \mathrm{rad}$ the average velocity, $\dot{y}_{s r}$ passes through zero and attains its greatest value on a certain curve and then decreases by a curvilinear law and approaches zero at $\varphi_{0}=1.571$. the second type of graphs corresponds to the case when $\lambda>1$. In this case the greatest value of average velocity and the value of $\varphi_{0}$ corresponding to the maximum incerase, while the second part in which $\dot{y}_{s r}$ decreases is reduced and ceases to exist when $\lambda=4$. The third type of curves corresponds to the case when $\lambda<1$. In this case the average velocities only have negative values. Take note that as $\varphi_{0}$ increases these values smoothly decrease to miimum values and then smoothly increase and as $\lambda$ decreases the peak of the average velocity if observed at the smallest value of $\varphi_{0}$, while the section of the graph corresponding to the increase of $\dot{y}_{s r}$ is reduced. 
Fig. 6 shows the graphs of average vertical velocities of the ornithopter for different values of $w$. Three sections can be singled out on these graphs, which differ for positive and negative values of $w$. In the first case $($ for $w<0$ ) the average velocity at first increases almost proportionally to the amplitude of the links' oscillations, $\varphi_{0}$, attains the greatest value, then decreases also proportionally to the increase in $\varphi_{0}$, attais the least values and again increases. The second section of the graph is reduced as the value of $w$ increases and the repeated increase in the average velocity becomes less noticiable. The second type of graphs holds for $w>0$, on which an insignificant decrease in the average vertical velocity is observed as $\varphi_{0}$ increases and the greater the value of $w$, the more significant the decrease of $\dot{y}_{s r}$. The average velocity increases proportionally to $\varphi_{0}$ on the graphs up to the maximum value and after that $\dot{y}_{s r}$ decreases and the value of the last section and the value by which the average velocity decreases both decrease as $w$ increases. The graph for $w=0$ is a transition between the graphs for $w<0$ and $w>0$.

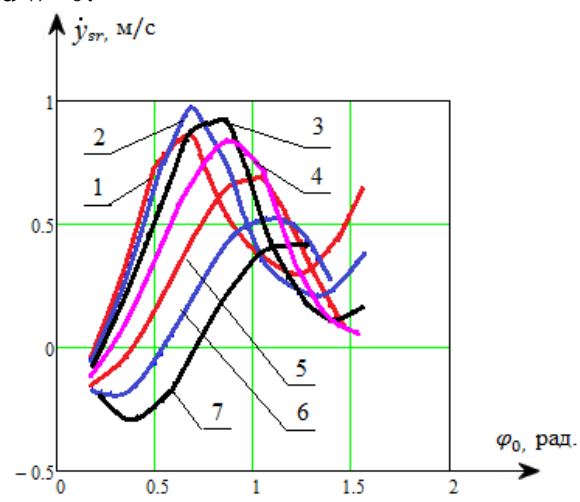

Fig. 6. Graphs of $\dot{y}_{s r}\left(\varphi_{0}\right)$ for $\lambda=1, w=0 \mathrm{rad}, T=0,03 \mathrm{~s}$ : $1-w=-15^{0}, 2-w=-10^{0}, 3-w=-5^{0}, 4-w=0^{0}, 5-w=5^{0}$, $6-w=10^{0}, 7-w=15^{0}$.

\section{Conclusion}

In this article we presented the results of the study of the vertical flight of a five-link robot-ornithopter with flapping folding wings whose main distinctive feature is that the motion of its wings imitates the flaps of a seagull. A mathematical model of the flight of the device in the vertical plane was developed and presented in matrix form. We discussed in detail the interaction of the links of the device with the air enviroment, determined the positions and velocities of the points of application of reduced aerodynamic forces to each link of the robot.

Numerical modelling of the robot's flight was conducted based on the mathematical model and three modes of flight: descent, hovering and ascet were established based on the time graphs of of the vertical coordinate of the body's center of mass. Hovering is observed for each value of $\varphi_{0}$ only at a given value of $\omega$, while ascent and descent have a wide range of variation of the two stated parameters.

The graphs of the average values of the body's vertical velocities against the amplitudes of the links' oscillations were constructed and the influence of the osillation parameters: amplitude ratios of the oscillations of links 1 and 2 (4 and 5) and the displacement, $w$ of the equillibrium position of the oscillations from zero on the alternation sequence of the flight modes, maximum and minimum values of $\dot{y}_{s r}$.

The study was conducted with the financial support of RFBR under scientific project № 16-08-00787.

\section{References}

1. J.D. DeLaurier, Canadian aeronautics and space journal, 40, 1, 10-18 (1994)

2. A.N. Brooks, AIAA, 85, 1446 (1985)

3. J.D. DeLaurier, The aeronautical journal of the royal aeronautical society, 153-161 (1993)

4. E. Craparo, B.A Ingram, 41st aerospace sciences meeting and exhibit, p. 1-9 (2003)

5. S.F. Jatsun, L.Yu. Vorochaeva, S.V, Vibroengineering PROCEDIA, 8, $74-79$ (2016)

6. Z.J. Jackowski, Diss. Massachusetts Institute of Technology (2009)

7. J. Maglasang, K. Isogai, N. Goto, M. Yamasaki, Memoirs of the Faculty of Engineering, 66, 1, 71-82 (2006) 